\title{
BARRERAS IDENTIFICADAS POR EL PERSONAL DE SALUD PARA EL TAMIZAJE DEL VIRUS DE INMUNODEFICIENCIA HUMANA EN POBLACIÓN INDÍGENA DE LA AMAZONÍA PERUANA
}

\author{
Helen Palma-Pinedo ${ }^{1, a}$, Mary F. Reyes-Vega ${ }^{2, b}$
}

\begin{abstract}
RESUMEN
Objetivos. Describir las barreras identificadas por el personal de salud para el tamizaje de virus de inmunodeficiencia humana (VIH) a población indígena en establecimientos de salud de cuatro regiones de la Amazonía peruana. Materiales y métodos. Estudio mixto. La fase cualitativa fue de tipo exploratoria y la fase cuantitativa se basó en un estudio descriptivo transversal de carácter exploratorio. Se seleccionaron 87 establecimientos en los que se aplicó una encuesta, además se realizaron 51 entrevistas a personal de salud de algunos de los establecimientos. Las entrevistas fueron codificadas mediante el software Atlas.ti 7.0. Para las encuestas se realizó un análisis estadístico descriptivo. Resultados. Las barreras identificadas fueron geográficas, socioculturales, y vinculadas al sistema de salud. Fue más frecuente encontrar desabastecimiento de insumos o reactivos en puestos de salud $(40,0 \%)$ en comparación con los centros de salud $(25,0 \%)$ y hospitales $(11,1 \%)$. El principal aspecto que limitó la realización de campañas de tamizaje para el acceso a la población indígena fue la falta de presupuesto $(59,3 \%)$, la falta de recursos humanos, el que las personas no acepten la toma de muestra de sangre, así como la falta de abastecimiento de insumos o reactivos para las pruebas de diagnóstico. Conclusiones. Las principales barreras identificadas por el personal de salud son en su mayoría las vinculadas al sistema de salud y de índole sociocultural. Se recomienda considerarlas para la mejora de la oferta de los servicios, así como para su adecuación cultural.
\end{abstract}

Palabras clave: VIH; Población Indígena; Tamizaje Masivo; Perú (fuente: DeCS BIREME).

\section{BARRIERS IDENTIFIED BY THE HEALTH STAFF FOR THE SCREENING OF HUMAN IMMUNODEFICIENCY VIRUS IN INDIGENOUS POPULATIONS OF THE PERUVIAN AMAZON}

\begin{abstract}
Objective. To describe the barriers identified by the health staff for the screening of human immunodeficiency virus (HIV) in indigenous populations at health centers located in four regions of the Peruvian Amazon. Materials and Methods. Mixed study. The qualitative phase was exploratory and the quantitative phase was based on a cross-sectional descriptive study of exploratory character. Eighty-seven (87) facilities were selected and a survey was applied. Additionally, 51 interviews were conducted to the health staff of some of the establishments. The interviews were codified by means of software Atlas.ti 7.0. For the surveys a descriptive statistical analysis was made. Results. The barriers identified were geographic, sociocultural, and others linked to the health system. It was more frequent to find shortage of inputs or reagents in health centers $(40.0 \%)$ as compared to clinics and $(25.0 \%)$ and hospitals $(11.1 \%)$. The main aspect that limited the screening campaigns for indigenous population was the lack of budget (59.3\%), the lack of human resources, people not accepting to have blood samples taken, as well as the lack of supplies or reagents for the diagnostic tests. Conclusions. The main barriers identified by the health staff are mostly those linked to the health system and those of a sociocultural nature. We recommend considering them for the improvement of the supply of the services, as well as for its cultural adjustment.
\end{abstract}

Keywords: HIV; Indigenous Population; Mass Screening, Peru (source: MeSH NLM).

El artículo es parte de la tesis de Helen Palma-Pinedo para obtener el grado de magíster en Salud Pública de la Universidad Nacional Mayor de San Marcos.

1 Universidad Nacional Mayor de San Marcos. Lima, Perú.

Centro Nacional de Epidemiología, Prevención y Control de Enfermedades, Ministerio de Salud. Lima, Perú

Antropóloga, magíster en Salud Pública; ${ }^{\mathrm{b}}$ médico cirujano, magíster en Epidemiología.

Recibido: 31/07/2018 Aprobado: 21/11/2018 En línea: 21/12/2018

Citar como: Palma-Pinedo H, Reyes-Vega M. Barreras identificadas por el personal de salud para el tamizaje de VIH en población indígena de la Amazonía peruana. Rev Peru Med Exp Salud Publica. 2018;35(4):610-9. doi:10.17843/rpmesp.2018.354.3855. 


\section{INTRODUCCIÓN}

Se estima que, en el 2017, 36,9 millones de personas vivían con el virus de la inmunodeficiencia humana $(\mathrm{VIH})$ en todo el mundo ${ }^{(1)}$. Si bien se observan avances en la lucha contra la epidemia a nivel mundial, este no suele ser homogéneo a nivel de todos los países e incluso a nivel subnacional, por ello, su morbilidad varía considerablemente entre países y regiones. En Perú, la epidemia es de tipo concentrada, con una prevalencia estimada en $0,3 \%$ en población general y una mayor prevalencia en las poblaciones expuestas a mayor riesgo, como los hombres que tienen sexo con hombres $(\mathrm{HSH})$ y las mujeres transgénero. A nivel nacional, las regiones más afectadas son aquellas más densamente pobladas de la costa y selva del país ${ }^{(2)}$.

Las poblaciones indígenas amazónicas son grupos que históricamente se han encontrado en un mayor estado de vulnerabilidad debido a la exclusión e inequidad visible en sus condiciones socioeconómicas, dificultades para el acceso a servicios de salud para la detección, el tratamiento y la prevención del $\mathrm{VIH}$ y otras enfermedades ${ }^{(3,4)}$. Existen estudios que señalan que las tasas de $\mathrm{VIH}$ y síndrome de inmunodeficiencia adquirida $(\mathrm{VIH} / \mathrm{Sida})$ son más altas en esta población que en la población general, afectando especialmente a los más jóvenes y a las mujeres ${ }^{(5,6)}$. Estudios coinciden en señalar que su progresión en estas poblaciones podría ser más rápida que lo observado en las áreas urbanas ${ }^{(7,8)}$.

La información disponible sobre la prevalencia del VIH e infecciones de transmisión sexual en poblaciones indígenas del Perú no es abundante. Un estudio publicado el 2007 realizado entre los Chayahuita en Loreto, halló una prevalencia de $7,5 \%$ y de $6,3 \%$ para $\mathrm{VIH}$ y sífilis, respectivamente. Ninguno de los adultos participantes manifestó haber usado condón alguna vez ${ }^{(6)}$. Otro estudio publicado el 2008 realizado en comunidades indígenas de Loreto, estimó una prevalencia de $\mathrm{VIH}$ de $0,7 \%$ de casos correspondientes a $\mathrm{HSH}^{\left({ }^{9}\right)}$. Asimismo, un estudio realizado por la Dirección General de Epidemiología del Ministerio de Salud del Perú en mujeres embarazadas y sus parejas sexuales masculinas en seis comunidades indígenas amazónicas de Loreto, Ucayali, Cusco, Junín, y Pasco, evidenció una alta prevalencia de hepatitis B y sífilis transmitidas principalmente por vía sexual que se presentaron de manera hiperendémica y endémica, respectivamente. La prevalencia de $\mathrm{VIH}$ en las mujeres embarazadas fue de $0,16 \%$ y en sus parejas varones $0,29 \%^{(7)}$.

Según la Organización Mundial de la Salud, las pruebas de $\mathrm{VIH}$ y la consejería constituyen un punto de entrada decisivo y esencial para la prevención y tratamiento ${ }^{(10)}$. La accesibilidad a las pruebas resulta fundamental para lograr un tratamiento oportuno. En el Perú, desde 1997

\section{MENSAJES CLAVE}

Motivación para realizar el estudio. Son varios los documentos de envergadura nacional e internacional que resaltan la importancia del fortalecimiento de las medidas de prevención y control del virus de inmunodeficiencia humana (VIH), entre las que destaca la ampliación de la cobertura del tamizaje sobre todo en poblaciones clave y vulnerables como la población indígena, lo que permitirá brindar un tratamiento oportuno.

Principales hallazgos. La presente investigación identificó que existen barreras reconocidas por el personal de salud que son de tipo geográficas, socioculturales, y vinculadas al sistema de salud.

Implicancias. Conocer estas limitaciones permitirá dirigir los esfuerzos y focalizar los recursos para contribuir a la disminución de la incidencia del VIH en la población indígena del país.

se aprobaron normas destinadas a la prevención de las ITS y del VIH/Sida. Se han logrado avances, sin embargo, aún existen brechas importantes en las coberturas de diagnóstico y tratamiento, siendo estas mayores en el caso de población indígena.

Por ello, el objetivo de este estudio fue identificar las barreras que afectan el desarrollo del tamizaje para $\mathrm{VIH}$ en población indígena de la Amazonía del Perú, a fin de focalizar los recursos en disminuir dichas barreras, y así contribuir a la disminución de la incidencia de VIH en la población indígena del país.

\section{MATERIALES Y MÉTODOS}

Se realizó un estudio mixto, en el cual la fase cualitativa tuvo una orientación exploratoria mientras que la fase cuantitativa se basó en un estudio descriptivo transversal de carácter exploratorio. La recolección de datos se hizo en dos fases que se desarrollaron de manera secuencial durante el periodo setiembre-diciembre 2014, en establecimientos de salud (EESS) de las regiones Loreto, Madre de Dios, Huánuco y Amazonas.

\section{FASE CUALITATIVA}

La información fue obtenida a partir de 51 entrevistas semiestructuradas. Los criterios de selección de los entrevistados fueron: personal de salud de establecimientos (puestos de salud, centros de salud, hospitales) ubicados en las cuatro regiones seleccionadas, personal responsable de Epidemiología de la Estrategia para la Prevención y Control de ITS, VIH y Sida (ESNITSS), y de personal de laboratorio. Estos criterios fueron definidos con el objetivo de llegar al personal más idóneo al tema de esta investigación. Del total de entrevistados, 27 fueron mujeres y 24 hombres; además nueve pertenecían a una Dirección Regional de 
Salud (DIRESA), 12 a una red de salud, 18 a una microrred, y 12 a un puesto de salud.

La guía de entrevista presentó preguntas en torno a tres dimensiones: barreras geográficas, barreras socioculturales, y barreras del sistema de salud. La guía fue sometida para su validación a juicio de expertos (cinco en total) con amplio conocimiento en metodología de investigación, VIH/Sida, y/o población indígena. Las entrevistas fueron realizadas en las horas y lugares elegidos por los informantes para su comodidad, las cuales en todos los casos no tuvieron una duración menor a los 30 minutos. Las entrevistas fueron grabadas, transcritas, y codificadas mediante el programa Atlas.ti 7.0 a partir de un análisis temático; lo que permitió la identificación de patrones, así como la relación entre conceptos y procesos descritos por los entrevistados.

\section{FASE CUANTITATIVA}

Tuvo el objetivo de conocer la amplitud de las barreras identificadas en la fase exploratoria. Con ese fin, se elaboró una encuesta para ser aplicada en todos los EESS de la Amazonía, en el ámbito geográfico de las cuatro regiones seleccionadas, que cumplieran los siguientes dos criterios de inclusión: EESS que hayan notificado casos de VIH y/o Sida en el periodo 2010-2014, y EESS que atendían a población indígena amazónica, considerando a los establecimientos de primer nivel de atención con población indígena asignada y los hospitales de referencia regionales, los cuales eventualmente podían recibir un caso referido proveniente de una población indígena.

Participaron todos los EESS de cada DIRESA que cumplieron con los criterios de inclusión antes mencionados, siendo un total de 87 establecimientos. De estos, se excluyó a seis debido a que las encuestas no fueron llenadas en la mayoría de ítems. Así, participaron del estudio 81 EESS, de los cuales $40,7 \%$ correspondieron a la región Loreto, 38,3\% a Amazonas, $12,3 \%$ a Madre de Dios y $8,6 \%$ a Huánuco. El $45,7 \%$ de los establecimientos eran puestos de salud, el $42,0 \%$ eran centros de salud y el $12,3 \%$ eran hospitales.

La encuesta fue autoaplicada entre el personal de la Estrategia de Prevención y Control de las ITS, VIH y Sida, conjuntamente con el personal de epidemiología de los EESS seleccionados. Se seleccionó a dicho personal por ser aquellos responsables de implementar las acciones de tamizaje para $\mathrm{VIH}$, así como realizar la vigilancia epidemiológica de la infección por VIH en la población de su jurisdicción. El personal de vigilancia epidemiológica de la DIRESA, previamente capacitados, realizó el control de calidad de las encuestas.

La validación de la encuesta fue realizada mediante apreciación de juicio de expertos (cinco), siendo personas que habían realizado investigaciones relacionadas a VIH en poblaciones indígenas, personas cuya actividad laboral está vinculada a este tema o expertos en metodología de la investigación y validación de instrumentos. Una vez incorporadas las recomendaciones se procedió a la realización de una prueba piloto en 24 establecimientos que atienden a pacientes con $\mathrm{VIH}$ procedentes de comunidades indígenas amazónicas de las regiones de San Martín y Ucayali.

El instrumento recolectó información sobre variables categóricas y cuantitativas para las cuales se realizó un análisis estadístico descriptivo, con medidas de frecuencia y medidas de tendencia central.

La presente investigación fue aprobada para su ejecución por la DIRESA de cada región seleccionada, con las cuales se coordinó para su ejecución. A su vez, fue aprobada por el Comité de Ética del Instituto de Medicina Tropical «Daniel Alcides Carrión», de la Universidad Nacional Mayor de San Marcos.

\section{RESULTADOS}

\section{RESULTADOS DEL ANÁLISIS CUALITATIVO}

\section{Barreras geográficas}

Como barreras geográficas se hace alusión a las dificultades para el acceso debido a las distancias geográficas. El personal entrevistado identificó a este punto como una de las principales barreras. La dispersión de la población hace que el acercamiento del personal de salud para el tamizaje se limite por la demanda en tiempo y recursos. De igual manera, se dijo que resulta costoso para la población acercarse a los EESS, sobre todo a los de mayor capacidad resolutiva.

"La barrera es la lejanía, viven tan dispersos que para que vengan el costo es alto, dónde se quedan aquí, dónde comen, a veces no tienen familiares» (ESNITSS y TARGA-Hospital Santa Gema-Loreto).

"Ellos vienen a su hotel y la comunidad donde yo trabajo es de bajos recursos, si les dices que regresen mañana ellos tienen que gastar un día más, a veces ellos vienen solo programándose un día de estadía. Más es eso la dificultad» (Puesto de Salud Vuelta Grande-Microrred Laberinto-Madre de Dios).

Por otro lado, la lejanía geográfica tiene consecuencia también en el acceso a la prueba y a otros exámenes necesarios para atender los casos confirmados de $\mathrm{VIH}$.

«El acceso a la prueba es una brecha importante. Entonces hay también una serie de exámenes de laboratorio que hay que tomar como el perfil hepático, pero en Nieva no hay, eso hay que ver con laboratorio, cuáles son los requisitos básicos antes de iniciar el tratamiento, como una prueba de hemoglobina porque el tratamiento da anemia. Tengo que saber si tiene hepatitis, sino no le puedo dar» (ESNITSS-Red Bagua-Amazonas). 
La consecuencia de esta barrera es la búsqueda limitada de casos en estos ámbitos, lo que conlleva a un subregistro.

"Como VIH hemos tenido una curva que se ha incrementado por años, incluso tenemos ya casos en comunidades indígenas lo que es preocupante, hasta fallecidos. Es también porque se está buscando más, hemos hecho un tamizaje en Andoas el año pasado y salieron como 12 casos de VIH confirmados por Western Blott, eran pacientes quechuas de los cuales ya han fallecido dos. Entonces se incrementan los casos porque se está buscando. Pero no podemos entrar así nomás hay que hacer toda una consulta, también depende del presupuesto» (Epidemiología-Red Datem del Marañón-Loreto).

\section{Barreras socioculturales}

Con estas barreras se hace referencia a aquellas características propias del entorno o vinculadas a la población que, desde el punto de vista del personal, dificulta su acercamiento para el tamizaje.

\section{Presencia de actividades ilegales:}

La presencia de actividades ilegales como la minería, tala no autorizada, y el trabajo sexual, fue mencionada como una barrera que forma parte del contexto social al que pertenecen algunos sectores de la población indígena que ellos deben atender. Esto se presentó de manera más recurrente en las regiones Madre de Dios y Amazonas, en los que varones y mujeres indígenas participan de estas actividades.

«Aquí se presentan dos problemáticas: la minería informal y la prostitución. Con la minería se generan los bares, el trabajo sexual, y ahí se da el problema. Es justamente esa población que se dedica a la minería la que se rehúsa a hacerse la prueba y recibir atención» (ESNITSS- DIRESA Madre de Dios).

La principal dificultad es lograr el acercamiento a estos grupos que viven subordinados a un patrón o jefe que, dada la actividad que realizan, evitan cualquier tipo de acercamiento por parte de actores del Estado. Este punto representa además un reto para el manejo de la confidencialidad ya que, como se aprecia en las citas de abajo, se exige en algunos casos conocer los resultados del tamizaje.

"Para que las trabajadoras sexuales se tamicen primero tiene que aceptar el dueño del bar, y te dicen 'si vas a tamizar está bien, pero me tienes que decir cuál es el resultado para saber', y como sabemos eso está prohibido por la Ley Contrasida» (Salud Pública-Red Bagua).

"Hay chicas que no quieren también, o tenemos que coordinar con los presidentes de los bares, son como una asociación tienen su presidente, tesorero. En San Juan son 28 bares, entonces coordinamos previamente con el presidente, le decimos 'vamos a hacer una charla y tomar muestra', pero hay chicas que no quieren.

\section{E: ¿Estos dueños les exigen conocer los resultados?}

De los 28 unos 10, tratamos de explicarles que el resultado es personal, pero dicen 'queremos saber para conocer con qué chicas estamos trabajando'» (Puesto de Salud San Juan GrandeMicrorred Colorado-Madre de Dios).
Presencia de población indígena «golondrina» o transeúnte:

La población denominada por los entrevistados como "golondrina» o transeúnte se encuentra presente en ámbitos con actividades económicas marcadas como la minería, tala, comercio, o en zonas de carretera en las cuales la mayoría de población permanece en dichos lugares de manera temporal. Este fenómeno se daba, por ejemplo, en Madre de Dios.

"La población que tenemos acá es generalmente de la zona minera, entonces la gente migra de diferentes ciudades precisamente por la minería, trabaja en La Pampa, Hueipetuhe, entonces la gente que está vienen de Puno, Abancay, Cuzco, Arequipa, Lima» (Hospital Santa Rosa-Madre de Dios).

Esto se presentó como una barrera en tanto representa una población difícil de captar y retener, más aún si el resultado del tamizaje es positivo y se tiene la necesidad de hacer otros exámenes, así como para el tratamiento.

«A diferencia de otras regiones en Madre de Dios hay mucha gente transeúnte, gente que viene y se va. Entonces muchos de los pacientes notificados no los tenemos acá, se van a otros sitios, $y$ es difícil captarlos para el tamizaje» (ESNITSS-DIRESA Madre de Dios).

\section{Estigma y discriminación:}

El personal entrevistado manifestó que la discriminación y estigma asociados al VIH afecta el tamizaje en tanto el virus es identificado como la consecuencia de un comportamiento homosexual, lo que genera rechazo en algunas comunidades y lleva incluso a expulsiones como ha sido registrado en el caso del pueblo awajún presente en Amazonas y Loreto.

«Al principio teníamos mucha dificultad incluso para las charlas, no nos aceptaban. Pensaban que quien escuchaba era porque tenía la enfermedad, pero ahora eso ha mejorado, conversamos con los apus para que ellos hablen con su gente. Felizmente la gente ya se está quitando de la cabeza que es sólo el HSH el que tiene la enfermedad, se creía que todo homosexual tenía VIH y no es así. Los HSH se han concentrado aquí porque se van de sus pueblos o los expulsan porque los ven amanerados» (ESNITSS-Red Datem del Marañón-Loreto).

Esta barrera también presenta retos para el manejo de la confidencialidad según indica la Norma Técnica ${ }^{(11)}$, dado que en algunas comunidades indígenas es el apu (líder) quien representa la autoridad y quien en ocasiones exige conocer los resultados del tamizaje para expulsar a los casos positivos.

\section{«E: ¿Qué tipo de barreras se presentan?}

De todo tipo, pero las logísticas son las menos creo. Pero sí las barreras culturales son extremadamente marcadas. Por ejemplo, ayer escuchaba que un dirigente nativo decía 'acá todo se sabe, entre nosotros no hay secretos, en el pueblo indígena no hay secretos'. Primero que hay una ley de confidencialidad, pero en el caso de ellos es el apu el que decide. Si uno va y hace una campaña de salud, el apu quiere ver los resultados. Entonces eso nos genera problemas, porque convencerlos es difícil, y después 
que estén señalando quién tiene Sida y quién no, porque incluso en algunos casos los botan de la comunidad. Eso es algo bastante traumático, imagínese el hecho de darle los resultados al apu, estaríamos yendo en contra de la ética y lo que dice la Norma» (Epidemiología-DIRESAAmazonas).

Pero, además, la confidencialidad es un aspecto que también debería ser mejor llevado por el personal que desarrolla el tamizaje de manera intramural.

«Entonces para nosotros sobre todo que somos hospital es difícil salir, pero los que vienen acá no quieren ser abordados por otra gente, pero también hay que preparar y concientizar al personal de salud de los establecimientos, porque a veces cometen errores en la confidencialidad, hablan de más a otras personas y la gente se incomoda y ya no quiere venir» (Hospital Santa Rosa-Madre de Dios).

\section{Idioma:}

Que el personal no maneje a profundidad el idioma de la población es una barrera que afecta la comunicación entre el personal de salud y los pacientes indígenas, dificultando la comprensión de aspectos importantes como qué es el $\mathrm{VIH}$, la importancia del tamizaje, el consentimiento informado, entre otros.

"Una dificultad es el idioma, hay que respetar los aspectos interculturales. Estoy viendo como introducimos dentro de la capacitación estos temas, decir que el personal tiene que consultar con el apu en su idioma antes de sacar las pruebas, cómo comunicar, sensibilizar, para facilitar la extracción de sangre» (ESNITSS-DIRESA Loreto)

\section{Consentimiento para el tamizaje:}

Otra barrera mencionada por el personal de salud fue vinculada al procedimiento para obtener el consentimiento para realizar el tamizaje. En muchas comunidades indígenas amazónicas se exige pasar primero por un proceso de aprobación por parte de sus líderes y/o la Junta Directiva, este proceso es conocido como consulta.

«Al trabajar con poblaciones indígenas hay que entrar con consulta previa, hacer varias conversaciones, no podemos entrar de frente. Ahí tenemos que aprovechar no sólo para ver hepatitis, sino también VIH, sífilis, se dan charlas, hay que hacer el consentimiento» (ESNITSS-Red Datem del Marañón-Loreto).

Este proceso exige voluntad de diálogo por parte del personal de salud, pero también mayores recursos porque exigen más tiempo que los procedimientos que se siguen en contextos urbanos con población mestiza. El no hacerlo, implica asumir un riesgo de futuros conflictos.

"Cuando hay problemas es cuando no se coordina, inclusive puede haber un conflicto y hay problemas. Por eso cuando vamos a tamizar vamos convocando a sus dirigentes. Primero vamos a coordinar, acordamos el día, luego el 'apu' tiene que comunicar, también hacemos reunión con la gente misma, luego tenemos que ir casa por casa y preguntar y ahi que acepten. Todo eso implica mayor inversión, combustible, viáticos, etc.» (Epidemiología-Red Datem del Marañón-Loreto).

\section{Desconfianza por el proceso de tamizaje:}

El personal de salud identificó como una barrera la desconfianza al tamizaje, debido al hecho que existiría la percepción extendida de que la sangre extraída serviría para ser vendida. Esto se presentó en Amazonas, Huánuco y Loreto.

«Pero también es el tamizaje, no quieren que se les saque sangre, piensan que uno lo va a vender o hacer algo» (Salud Pública-Red Bagua).

«Para la toma de muestras la población es medio reacia. Nosotros tomamos del dedo porque si tomamos de la vena ellos todavía tienen la creencia de que uno va a vender su sangre, y no se dejan. En cambio, en el dedo igual les rogamos pero se dejan, ya si hay que confirmar les explicamos y les sacamos de la vena (...) no sé quién les habrá metido la idea de que van a vender su sangre, pero así dicen» (Centro de Salud Yuyapichis-Microrred Puerto Inca-Huánuco).

La desconfianza también se relaciona con la cantidad de sangre que se extrae, mientras más cantidad de sangre mayor es la desconfianza y rechazo.

"Los indígenas tienen temor porque les sacan demasiada sangre $y$ dicen que se debilitan, y esa es la dificultad, que acepten sacarse la cantidad que necesitamos para hacer todos los exámenes que corresponde. Para eso necesitamos 3 tubos y ellos no aceptan, o se desaniman. Con eso sacamos varios exámenes y aparte para enviarlo para hacer CD4, carga viral, porque sacar en otra oportunidad es difícil»» (Laboratorio-Red Datem del Marañón-Loreto).

Por último, se mencionó que en algunas personas se presenta desconfianza debido a que no se comprende cómo puede obtenerse el resultado de manera tan rápida, lo que lleva a pensar que pueda ser erróneo.

"Uno dijo que esa prueba no valía, que cómo va a salir eso al instante, que él tenía su médico en su pueblo que lo iba a curar y se fue» (Puesto de Salud Vuelta Grande-Microrred Laberinto-Madre de Dios).

\section{Percepción del VIH como «daño»:}

El personal de salud entrevistado mencionó que sectores de la población indígena que atienden presentan una percepción extendida del VIH como «daño» (padecimiento vinculado a la brujería), lo que hace que no se dé importancia a sus señales de alerta ni tampoco al tamizaje, lo que lleva a optar por los servicios de terapeutas de la medicina tradicional en lugar del personal de salud. Esto se presentó de manera más frecuente en Amazonas y Huánuco.

«Esta población no asume al VIH como un problema. La población migra, se esconde, no percibe la gravedad. Para ellos es 'daño' y aparecen cuando ya están en la etapa sida» (ESNITSS-Red Bagua-Amazonas). 
"Lo otro es que las concepciones mágico-religiosas son extremadamente fuertes. Esto no permite que crean en lo que nosotros les damos. Por ejemplo, uno hace un cuadro de Sida, está con diarreas, baja de peso, entonces le haces la prueba y le dices que salió positivo. Pero como no le ofrecíamos el tratamiento no creían, se iban con el brujo. Él les dice que es maleficio, daño, le da unas plantas y el paciente se mejora. Bueno, al final los productos químicos son muchas veces derivados de productos naturales, de plantas, y por eso será» (Epidemiología-DIRESAAmazonas).

«En un caso de VIH, ellos no pensaban que era eso, pensaban que era daño, un embrujo, por eso se iban por otras opciones. Pero la información sobre qué trata la enfermedad, cómo se contagia, consecuencias, les hemos dado, pero ellos no toman consciencia en que eso les puede dar» (Centro de Salud Yuyapichis-Microrred Puerto Inca-Huánuco).

\section{Sexo del personal de salud y acceso a la población masculina:}

El género resulta una barrera en tanto un sector de los pacientes indígenas tiene preferencia por la atención hecha por personal del mismo sexo, de lo contrario se presenta un rechazo o incomodidad de su parte.

«A veces no se dejan examinar mucho, prefieren que les atienda una mujer, en el caso de los varones no quieren que las mujeres los atiendan. A veces como la comunicación es difícil por todo esto hay un rechazo a las muestras» (ESNITSS-Red Datem del Marañón-Loreto).

Por el lado de los pacientes, los varones resultan ser el grupo más difícil de captar para el tamizaje por el temor a un resultado positivo. Un argumento que se suele presentar para el rechazo es que «son fieles» y por tanto hacer el tamizaje es en vano, dando así a entender que sólo aquellos que tienen un comportamiento promiscuo son propensos a contraer al virus.

"Los varones son los que más tienen temor, como esta es zona minera los varones tienden a tener sus escapadas y no quieren saber. Cuando vienen acá no hay problema, cuando salimos es más difícil, no quieren» (Centro de Salud Laberinto-Microrred Laberinto-Madre de Dios)

"Se capta acá y cuando salimos a hacer atenciones integrales. Por ejemplo, ahora el programa está priorizando varones, pero a veces no se llega a la meta, dicen que son sanos, son fieles y por eso no lo hacen» (Centro de Salud Yuyapichis-Microrred Puerto Inca-Huánuco).

\section{Barreras vinculadas a la oferta de salud}

\section{Escaso personal de salud con competencias para atención a comunidades indígenas:}

Se mencionó que, dadas las dificultades para la accesibilidad y las precarias condiciones de vida en las comunidades, el personal de salud presente es escaso y el personal especializado ausente.

"Tenemos 33 comunidades nativas y tenemos presencia en cerca de 17 de esas comunidades con puestos de salud. Lamentablemente la mayoría solo cuentan con personal técnico, inclusive tenemos establecimientos que están vacíos porque nadie quiere ir a trabajar ahí. En la provincia del Manu están las comunidades más alejadas con EESS, pero hay otras más dispersas, la más dispersa puede llegar hasta 10 días de viaje en río dependiendo de la época» (Salud de las Personas-DIRESA Madre de Dios).

Esto lleva a que el tamizaje se realice a partir de campañas de salud esporádicas, y con limitaciones para el desarrollo de pruebas de mayor complejidad.

"Lo que se está haciendo es tomar pruebas serológicas que se envían a Tarapoto. Solo contamos con un infectólogo y esa es una gran barrera porque todo demora más, lo ideal sería que cada tres meses sean evaluados y eso no se puede hacer» (ESNITSS-Red Datem del Marañón-Loreto).

\section{Rotación y multifuncionalidad del personal de salud:}

La rotación del personal resulta una barrera en tanto debilita las relaciones con la población de las comunidades, haciéndola más sensible a la desconfianza o pobre comunicación.

"La primera barrera en esta población awajún es por la rotación del personal. Renuncian a trabajar allí, están dos meses y se van» (Salud Pública-Red Bagua).

La multifuncionalidad es un problema frecuente en el personal que atiende en comunidades indígenas, lo que lleva a que actividades como el tamizaje de VIH no sean prioritarias, o no se realicen por la falta de recurso humano.

«En todo establecimiento el personal que trabajamos ocupamos como cinco áreas diferentes, y para hacer todo no alcanzamos. En otros puestos están dos o tres, pero yo estoy sólo y cuando salgo a hacer extramural a veces vienen niños a hacer controles y los pierdo» (Puesto de Salud Alto Kanampa-Microrred Nieva).

Falta de competencias interculturales en el personal de salud:

Otra barrera mencionada fue la falta de una formación intercultural del personal de salud que conlleva a dificultades para su adaptación, pero sobre todo a la comprensión de la cultura de la población que atiende.

"Debería haber una formación intercultural, debería darse al $100 \%$ acá. Cuando viene gente de otros sitios no entienden, no se adaptan. Y si no sabemos cómo es la convivencia de ellos es bien difícil que entiendan. Como le dije el $75 \%$ de nuestra población es indígena y si no se tiene una formación intercultural es bien difícil» (Epidemiología-Red Datem del Marañón-Loreto).

"Lo otro es que debemos tener gente, sobre todo nativa, que los entiendan. Yo puedo andar con un nativo y le puedo hablar si quiero todo el día y todos los días, pero ellos me escucharán aparentemente, pero del uno al diez probablemente me entiendan la mitad» (Epidemiología-DIRESAAmazonas).

Falta de recursos para el tamizaje:

Otra barrera indicada es la falta de recursos necesarios para la toma de muestras y su conservación, como láminas, 
cadena de frío, termos; además de recursos necesarios para la logística como combustible o una movilidad fluvial.

«Primero para su conservación, no tenemos láminas para sacar muestras y no contamos con cadena de frío, tampoco tenemos termos, sólo me prestan a veces» (Puesto de Salud Vuelta GrandeMicrorred Laberinto-Madre de Dios).

\section{RESULTADOS DEL ANÁLISIS CUANTITATIVO}

Se encontró que el 95,1\% de los establecimientos realizaba alguna prueba diagnóstica para $\mathrm{VIH}$. El 95,1\% realizaba prueba rápida, y el $6,2 \%$ realizaban ELISA, ningún establecimiento realizaba Western Blot. El 28,4\% presentaban «siempre» 0 «casisiempre» desabastecimiento de insumos para la realización de dichas pruebas. Fue más frecuente encontrar desabastecimiento en puestos de salud (40,0\%) en comparación con los centros de salud $(25,0 \%)$ y hospitales $(11,1 \%)$. Los establecimientos de Loreto y Amazonas reportaron presentar «siempre» o «casi siempre» desabastecimiento de insumos o reactivos para la realización de pruebas diagnósticas $(36,7 \%$ y $34,5 \%$, respectivamente) lo cual fue menor en otras regiones (Figura 1).

Al analizar los problemas en los EESS para realizar el diagnóstico de infección por $\mathrm{VIH}$, se observó que los más frecuentes fueron los resultados tardíos $(37,0 \%)$, así como, el hecho de que los pacientes no accedían a tomarse muestras de sangre $(37,0 \%)$ (Tabla 1$)$.

El $71,6 \%$ de los establecimientos realizaba campañas de tamizaje y/o búsqueda activa de casos de VIH/Sida en comunidades indígenas amazónicas, siendo con mayor frecuencia ejecutadas de forma trimestral o semestral (Tabla 1).

El principal aspecto que limitó la realización de campañas de tamizaje y el acceso de la población indígena a las

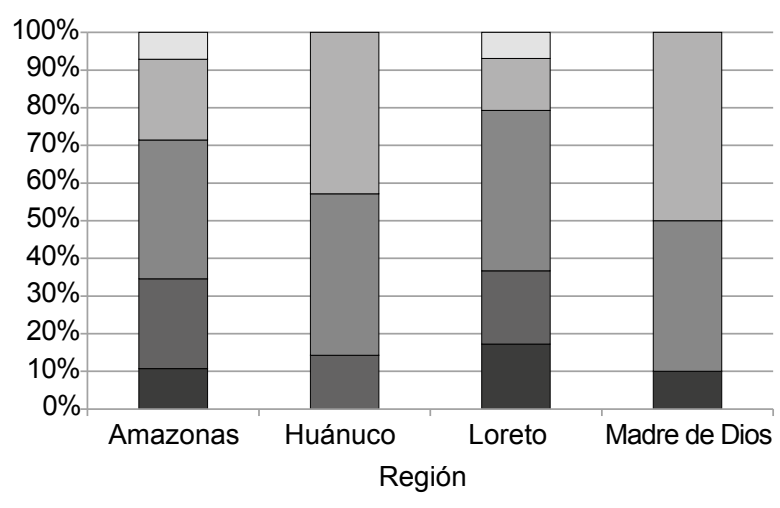

$\square$ Siempre $\square$ Casi siempre $\square$ A veces $\square$ Casi nunca $\square$ Nunca

Figura 1. Porcentaje del desabastecimiento de insumos o reactivos para la realización de pruebas diagnósticas según región. pruebas de tamizaje fue la falta de presupuesto (59,3\%); otras razones menos frecuentes fueron la falta de recursos humanos, el que los pacientes no accedan a que se les tome muestras de sangre, así como la falta de abastecimiento de insumos o reactivos para las pruebas de diagnóstico (Tabla 1).

Los establecimientos que reportaron tener mayores limitaciones de presupuesto fueron los de Madre de Dios y Huánuco; estos últimos fueron también los que reportaron mayor limitación de recursos humanos. En los establecimientos de Madre de Dios era mucho más frecuente que en otras regiones que los pacientes no accedan a la toma de muestras de sangre; mientras que, en los establecimientos de Amazonas, Huánuco y Loreto era frecuente el desabastecimiento de insumos o reactivos para la realización de pruebas diagnósticas para VIH (Tabla 2).

\section{DISCUSIÓN}

La presente investigación tuvo como resultado la identificación de barreras para el tamizaje a población indígena amazónica en el Perú, lo cual coincide con los resultados de otros estudios y documentos similares. Así, la dificultad para la accesibilidad en términos geográficos es presentada como una barrera importante para la atención en otros trabajos $(12,13)$, al igual que las barreras socioculturales ${ }^{(14)}$. Por otro lado, en un estudio cualitativo desarrollado con población indígena en Australia ${ }^{(15)}$, se identificó a las normas culturales de género como importantes para este grupo, ya que exigían ser atendidos por alguien de su mismo sexo lo cual no era ofrecido por los establecimientos por la falta de personal, lo que viene a ser un hallazgo similar a esta investigación. Por ello, una conclusión es la necesidad de contar con personal de ambos sexos en estos contextos como parte de una atención diferenciada que permita lograr un mejor acercamiento y atención a los pacientes.

El manejo de la confidencialidad también se presentó como un reto a afrontar en poblaciones indígenas de las regiones estudiadas. Al respecto, un estudio de Canadá ${ }^{(13)}$ menciona las dificultades sobre este punto en comunidades pequeñas, lo que tenía como consecuencia el rechazo a la prueba por el temor a que otros tengan conocimiento de ello. Esto es similar a lo encontrado en este estudio, a lo que se suma la exigencia de otras personas a conocer los resultados. En ese sentido, es necesario asegurar el cumplimiento de las normas vinculadas a la confidencialidad, así como sensibilizar sobre su importancia.

Otro aspecto identificado fue la desconfianza hacia el tamizaje debido a la extracción de sangre. Este punto es también identificado en otro trabajo ${ }^{(12)}$ a partir de una experiencia de un tamizaje masivo de hepatitis $B$ realizado en comunidades indígenas de Loreto. Al respecto, 
Tabla 1. Características, problemas y limitaciones para realizar el diagnóstico de infección por VIH, campañas de tamizaje y/o búsqueda activa de casos en establecimientos de salud que atienden casos procedentes de comunidades indígenas amazónicas a .

\begin{tabular}{|c|c|c|}
\hline Característica & Frecuencia & $\%$ \\
\hline \multicolumn{3}{|l|}{ Problemas para realizar el diagnóstico de infección VIH } \\
\hline Resultados tardíos & 30 & 37,0 \\
\hline Pacientes no acceden a toma de muestra de sangre & 30 & 37,0 \\
\hline Desabastecimiento de insumos y/o reactivos & 23 & 28,4 \\
\hline Falta de capacitación del personal & 21 & 25,9 \\
\hline Problemas en la conservación y/o calidad de la muestra & 11 & 13,6 \\
\hline Personal no conoce o no entiende norma técnica & 9 & 11,1 \\
\hline \multicolumn{3}{|c|}{ Periodicidad de campañas de tamizaje y/o búsqueda activa de casos } \\
\hline Mensual & 6 & 7,4 \\
\hline Trimestral & 22 & 27,2 \\
\hline Semestral & 16 & 19,8 \\
\hline Anual & 4 & 4,9 \\
\hline A veces & 1 & 1,2 \\
\hline Otra periodicidad & 9 & 11,1 \\
\hline No se realiza búsqueda de casos & 23 & 28,4 \\
\hline \multicolumn{3}{|c|}{$\begin{array}{l}\text { Limitaciones para realizar campañas de tamizaje y para el acceso a las pruebas de tamizaje } \\
\text { para VIH/Sida }\end{array}$} \\
\hline Falta de presupuesto & 48 & 59,3 \\
\hline Falta de recursos humanos & 33 & 40,7 \\
\hline Pacientes no acceden a toma de muestras & 33 & 40,7 \\
\hline Falta de abastecimiento de insumos o reactivos & 23 & 28,4 \\
\hline Problemas de accesibilidad geográfica & 1 & 1,2 \\
\hline Falta de tiempo del personal & 1 & 1,2 \\
\hline
\end{tabular}

VIH: virus de la inmunodeficiencia humana; Sida: síndrome de inmunodeficiencia adquirida.

a $\mathrm{Se}$ estudiaron 81 establecimientos de salud.

esta investigación tiene como resultado que un $37,0 \%$ del personal identificó como una limitación el hecho que los pacientes no accedieran a tomarse muestras de sangre. Sin embargo, en el caso del trabajo en mención, se identificó que esto se presentaba por la demora en la entrega de resultados, mientras que en nuestro estudio se debía a la idea de que su sangre sería comercializada. En todo caso, un punto común es la desconfianza frente a las estrategias provenientes desde el Estado, por lo que se hace importante respetar los procesos de consulta presentes en estos espacios a fin de recibir las autorizaciones y absolver dudas, así como cumplir con los compromisos asumidos.

En este trabajo se han identificado barreras vinculadas al sistema de salud. Ello coincide con, un estudio realizado en Francia ${ }^{(16)}$ que concluye en la relevancia de las características de la oferta para mejorar el acceso a la prueba. Por otro lado, en un documento de revisión realizado en Canadá ${ }^{(17)}$ se concluye que es el propio sistema de salud una de las partes responsables de los pobres resultados para superar este mal en la población indígena dado que no se han eliminado barreras que limitan su acceso a los programas de prevención y tratamiento. En ese sentido, antes de observar las barreras vinculadas a la población, es necesario que se aseguren las condiciones que permitan facilitar la atención. Por ejemplo, el Informe Defensorial $N^{\circ} 143^{(18)}$ en el cual se dan a conocer los resultados de una supervisión que incluye a Loreto; concluye que el $23 \%$ de establecimientos no contaban con pruebas rápidas para $\mathrm{VIH}$, y que el $20 \%$ no contaba con la infraestructura adecuada para brindar un servicio de consejería que garantice la privacidad y confidencialidad requeridas. Ello tiene que ver con lo encontrado en este trabajo, en la que se tiene como resultado que el $28,4 \%$ de establecimientos presentaban siempre o casi siempre desabastecimiento de insumos para la realización de dichas pruebas.

A partir de los resultados expuestos, se puede concluir en la necesidad de fortalecer una atención diferenciada con pertinencia cultural que permita mejorar las coberturas de tamizaje. Por otro lado, es necesario mejorar aspectos vinculados a la calidad de la oferta de servicios que atienden a estas poblaciones, que suelen ser los menos equipados y con menos personal. 
Tabla 2. Limitaciones según región para la realización de campañas de tamizaje y el acceso de la población indígena amazónica a las pruebas de tamizaje para VIH.

\begin{tabular}{|c|c|c|}
\hline $\begin{array}{l}\text { Limitaciones según } \\
\text { región }\end{array}$ & $\begin{array}{c}\text { Número de } \\
\text { establecimientos de } \\
\text { salud }^{\mathrm{a}}\end{array}$ & $\%$ \\
\hline \multicolumn{3}{|l|}{ Falta de presupuesto } \\
\hline Amazonas & $14 / 31$ & 45,1 \\
\hline Huánuco & $5 / 7$ & 71,4 \\
\hline Loreto & $21 / 33$ & 63,6 \\
\hline Madre de Dios & $8 / 10$ & 80,0 \\
\hline \multicolumn{3}{|c|}{ Falta de recursos humanos } \\
\hline Amazonas & $11 / 31$ & 35,5 \\
\hline Huánuco & $6 / 7$ & 85,7 \\
\hline Loreto & $13 / 33$ & 39,4 \\
\hline Madre de Dios & $3 / 10$ & 30,0 \\
\hline \multicolumn{3}{|c|}{$\begin{array}{l}\text { Pacientes no acceden a } \\
\text { tomarse muestras de sangre }\end{array}$} \\
\hline Amazonas & $12 / 31$ & 38,7 \\
\hline Huánuco & $2 / 7$ & 28,6 \\
\hline Loreto & $13 / 33$ & 39,4 \\
\hline Madre de Dios & $6 / 10$ & 60,0 \\
\hline \multicolumn{3}{|c|}{$\begin{array}{l}\text { Falta de abastecimiento } \\
\text { de insumos o reactivos }\end{array}$} \\
\hline Amazonas & $9 / 31$ & 29,0 \\
\hline Huánuco & $2 / 7$ & 28,6 \\
\hline Loreto & $10 / 33$ & 30,3 \\
\hline Madre de Dios & $2 / 10$ & 20,0 \\
\hline
\end{tabular}

a Se estudiaron 81 establecimientos de salud.
El presente estudio presenta la fortaleza de haber sido realizado con una metodología mixta lo que permitió profundizar en las reflexiones del personal de salud, así como medir la magnitud de las limitaciones inicialmente identificadas. Asu vez, tiene como limitaciones el no contar con la percepción de la población indígena respecto a la temática abordada, y el haber tenido un trabajo de campo de corta duración que no permitió profundizar en algunos aspectos.

Finalmente, se recomienda tomar en cuenta las barreras identificadas, particularmente las socioculturales con el fin de adecuar culturalmente las actividades vinculadas a la prevención y control del VIH, así como, las que corresponden a la oferta de servicios con el fin de mejorar el acceso a la prueba. Asimismo, se recomienda respetar los procesos de consulta presentes en las comunidades.

\begin{abstract}
Agradecimientos: al Centro Nacional de Epidemiología, Prevención y Control de Enfermedades. A Ronald Ayala Mendívil, asesor de la tesis en la que se basa este artículo. A los doctores Alicia Fernández Giusti, Eduardo Ticona Chávez, Luis Podestá Gavilano, y Luis Reátegui Guzmán, jurado que durante la defensa realizaron preguntas y comentarios muy pertinentes, además de recomendar su publicación.
\end{abstract}

Contribuciones de autoría: HPP y MRV participaron en la concepción, diseño y redacción del artículo.

Fuentes de financiamiento: Autofinanciado.

Conflictos de interés: las autoras declaran no tener conflictos de interés.

\section{REFERENCIAS BIBLIOGRÁFICAS}

1. ONUSIDA. Hoja Informativa: Día Mundial del SIDA, 2018 [Internet]. Ginebra: ONUSIDA; 2018 [citado el 14 de junio de 2018]. Disponible en: http://www.unaids.org/sites/default/ files/media_asset/UNAIDS_FactSheet_es.pdf

2. Ministerio de Salud del Perú, Dirección General de Epidemiología. Análisis de la Situación Epidemiológica del VIH/Sida en el Perú - 2013. Lima: Minsa; 2013.

3. Eyzaguirre Beltroy C, Falleque Solís C, Lou Alarcón S. Políticas para eliminar las barreras geográficas en salud. CIES/ Observatorio de la Salud. Lima: Consorcio de Investigación Económica y Social, Observatorio del Derecho a la Salud; 2007.

4. Portocarrero, J. El Estado frente a la salud de los pueblos indígenas. Intercambio. 2014;(26):26-8.
5. Bacon O, Pecoraro ML, Galvão J, PageShafer K. HIV/AIDS in Brazil. San Francisco: AIDS Policy Research Center, University of California; 2004.

6. Zavaleta $\mathrm{C}$, Fernández $\mathrm{C}$, Konda $\mathrm{K}$, Valderrama Y, Vermund SH, Gotuzzo E. High prevalence of HIV and syphilis in a remote native community of the Peruvian Amazon. Am J Trop Med Hyg. 2007;76(4):703-5.

7. Ormaeche M, Whittembury A, Pun M, Suárez-Ognio L. Hepatitis B virus, syphilis, and HIV seroprevalence in pregnant women and their male partners from six indigenous populations of the Peruvian Amazon Basin, 2007-2008. Int J Infect Dis. 2012;16(10):e724-30. doi: 10.1016/j.ijid.2012.05.1032.

8. Villalba JA, Bello G, Maes M, Sulbaran YF, Garzaro D, Loureiro CL, et al. HIV1 epidemic in Warao Amerindians from
Venezuela: spatial phylodynamics and epidemiological pattern. AIDS. 2013 Jul 17;27(11):1783-91. doi: 10.1097/ QAD.0b013e3283601bdb.

9. Bartlett EC, Zavaleta $\mathrm{C}$, Fernández $\mathrm{C}$, Razuri H, Vilcarromero S, Vermund $\mathrm{SH}$, et al. Expansion of HIV and syphilis into the Peruvian Amazon: a survey of four communities of an indigenous Amazonian ethnic group. Int J Infect Dis. 2008;12(6):e89-94. doi: 10.1016/j. ijid.2008.03.036.

10. Organización Mundial de la Salud. Documento normativo sobre las pruebas del VIH y la orientación: La OMS y el ONUSIDA reiteran su oposición a las pruebas obligatorias del VIH [Internet]. Ginebra: OMS; 2012 [citado el 12 de febrero de 2018]. Disponible en: http:// 
www.who.int/hiv/events/2012/world_ aids_day/hiv_testing_counselling/es/

11. Norma Técnica de Salud de Atención Integral del Adulto con Infección por el Vírus de la Inmunodeficiencia Humana (VIH). N097-MINSA/DGSP-V.02. (11 de diciembre del 2014).

12. Trujillo OV, Cabezas C, Marín L, Pari J, Salaverry O. Recolección de muestras sanguíneas en poblaciones indígenas. Rev Peru Med Exp Salud Pública. 2012;29(4):575-7. doi: http://dx.doi. org/10.17843/rpmesp.2012.294.408.

13. Tseng AL. Anonymous HIV testing in the Canadian aboriginal population. Can Fam Physician. 1996;42:1734-40.

14. Plan Estratégico Multisectorial de Prevención y Control de las ITS/VIHy SIDA,
2015 - 2019. Decreto Supremo No 035 2015-SA, Anexo. (23 de octubre del 2015.

15. Hengel B, Guy R, Garton L, Ward J, Rumbold A, Taylor-Thomson D, et al. Barriers and facilitators of sexually transmissible infection testing in remote Australian Aboriginal communities: results from the Sexually Transmitted Infections in Remote Communities, Improved and Enhanced Primary Health Care (STRIVE) Study. Sex Health. 2015;12(1):4-12. doi: 10.1071/SH14080.

16. d'Almeida KW, Pateron D, Kierzek G, Renaud B, Semaille C, de Truchis P, et al. Understanding providers' offering and patients' acceptance of HIV screening in emergency departments: a multilevel analysis. ANRS 95008, Paris, France. PLoS
One. 2013;8(4):e62686. doi: 10.1371/ journal.pone.0062686.

17. Public Health Agency of Canada [Internet]. Ottawa, Canada: Ministry of Health Canada; 2018 [citado el 12 de febrero de 2018]. Disponible en: http://www. phac-aspc.gc.ca/aids-sida/publication/ ps-pd/aboriginal-autochtones/chapterchapitre-4-eng.php

18. Defensoría del Pueblo. Fortaleciendo la respuesta frente a la epidemia del VIH/ Sida: Supervisión de los servicios de prevención, atención y tratamiento del VIH/Sida. Informe Defensorial $\mathrm{N}^{\circ} 143$. Lima: Defensoría del Pueblo; 2009.

Correspondencia: Helen Palma Pinedo Dirección: Calle Los Cóndores 284, Bellavista, Callao, Perú

Correo electrónico:helenpalmapinedo@hotmail.com

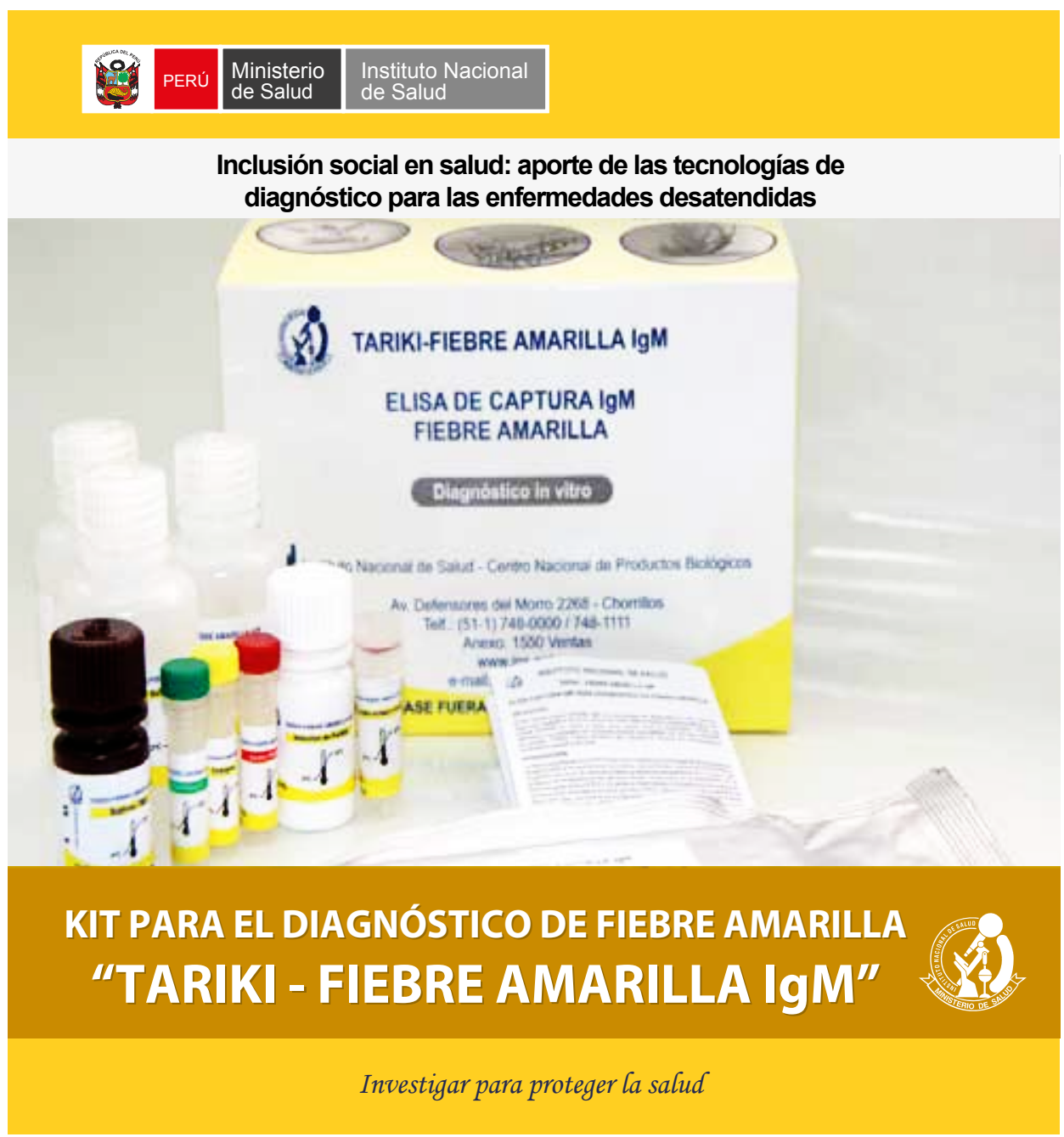

